Supporting information for

\title{
Gating of enhanced electron charging thresholds in self-assembled nanoparticle films
}

\author{
Yoshinori Suganuma and Al-Amin Dhirani
}

\begin{abstract}
Preparation of gold nanoparticles
$12.7 \mathrm{~mL}$ of $40 \mathrm{mM} \mathrm{HAuCl} / \mathrm{H}_{2} \mathrm{O}$ (yellow solution) was added to $25.6 \mathrm{~mL}$ of 50

$\mathrm{mM}$ tetraoctylammonium bromide (TOAB) in toluene. Aqueous and organic phases

became transparent and dark red, respectively, after a few seconds. The solution was vigorously stirred for $20 \mathrm{~min}$. Then, the aqueous phase was removed, and a reducing agent $\left(12.7 \mathrm{~mL}\right.$ of $\left.0.43 \mathrm{M} \mathrm{NaBH}_{4} / \mathrm{H}_{2} \mathrm{O}\right)$ was added. The organic phase became wine red. After vigorous stirring for $2 \mathrm{~h}$, the aqueous phase was removed, and the solution was washed with $30 \mathrm{~mL}$ of $0.1 \mathrm{M}$ sulfuric acid (3 times), then with $30 \mathrm{~mL}$ of $1 \mathrm{M}$ sodium carbonate ( 3 times), and finally $30 \mathrm{~mL}$ of $\mathrm{H}_{2} \mathrm{O}$ (3 times). The aqueous phase was removed, $\sim 10 \mathrm{~g}$ of Magnesium sulfate power was added, and then the power was removed by filtration. The final product solution of nanoparticles was stored in a refrigerator $\left(\sim 5^{\circ} \mathrm{C}\right)$ when not in use.
\end{abstract}

\section{Detailed experimental setups for conductance measurements \\ A home-made cryostat was used for low-temperature conductance measurements. Sample chips were placed on a copper stick covered with a thin Teflon sheet. Within a distance of a few cm, a GaAs diode temperature sensor (Scientific Instruments) and a tape heater were attached. The stick was inserted into a dewar and the edge of the stick was placed just above liquid nitrogen $\left(\mathrm{LiqN}_{2}\right)$. The distance between the sample chip and $\mathrm{LiqN}_{2}$ was within $5 \mathrm{~cm}$. The temperature was adjusted to $\pm 0.01 \mathrm{~K}$ with an SI9700 temperature controller (Scientific Instruments). Electrical contacts were made using thin copper magnet wires. Voltages were generated and read using a DAQ board (National Instruments) and LabView software (National Instruments). A home-made current- voltage converter (OPA128 or LF411) was used to measure current.}

\section{Control experiments for contact and edge effects}

To test whether gate effects are due to contact and edge effects at electrodes, we measured conductance maps using two samples with different central gate oxides thicknesses. Figures S1(a) and (b) show conductance maps as a function of bias and gate voltages at $77 \mathrm{~K}$. The sample in (a) was prepared using the same sample preparation procedures described in the main text. For the sample in (b), we did not etch the central part of the oxide so that the whole surface was covered with an oxide $\sim 300 \mathrm{~nm}$ thick. Both films had five exposures. Resistances of samples (a) and (b) at $270 \mathrm{~K}$ were $13 \mathrm{M} \Omega$ and $18 \mathrm{M} \Omega$, respectively. Electrodes were located about $0.5 \mathrm{~mm}$ away from the edge of the thin oxide ( 1 mm in width) in (a). Distance between electrodes for both samples (a) and (b) kept fixed ( 2mm). We observe much weaker gate effects for (b). This observation suggests that the gate effect is not due to contact and edge effects at the electrodes. 

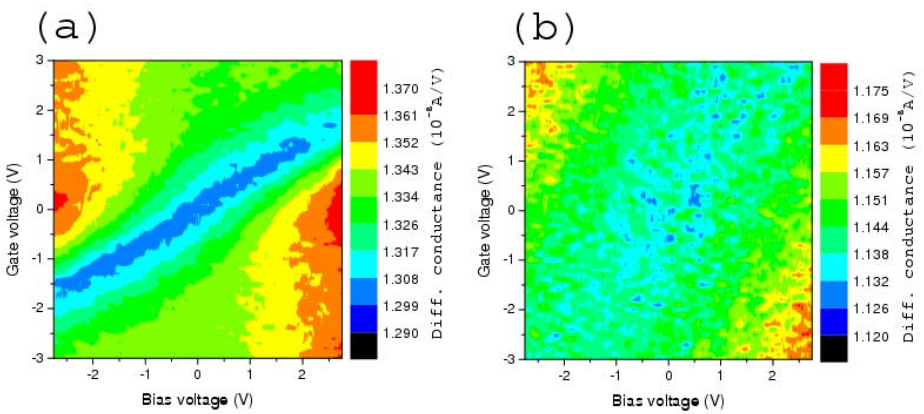

Fig.S1 